\title{
Comparison of Various Improved-Partition Fuzzy $c$-Means Clustering Algorithms in Fast Color Reduction
}

\author{
László Szilágyi ${ }^{1}$, Gellért Dénesi ${ }^{2}$, Levente Kovács ${ }^{3}$ and Sándor M. Szilágyi ${ }^{4}$
}

\begin{abstract}
This paper provides a comparative study of several enhanced versions of the fuzzy $c$-means clustering algorithm in an application of histogram-based image color reduction. A common preprocessing is performed before clustering, consisting of a preliminary color quantization, histogram extraction and selection of frequently occurring colors of the image. These selected colors will be clustered by tested $c$-means algorithms. Clustering is followed by another common step, which creates the output image. Besides conventional hard (HCM) and fuzzy $c$-means (FCM) clustering, the so-called generalized improved partition FCM algorithm, and several versions of the suppressed FCM (s-FCM) in its conventional and generalized form, are included in this study. Accuracy is measured as the average color difference between pixels of the input and output image, while efficiency is mostly characterized by the total runtime of the performed color reduction. Numerical evaluation found all enhanced FCM algorithms more accurate, and four out of seven enhanced algorithms faster than FCM. All tested algorithms can create reduced color images of acceptable quality.
\end{abstract}

\section{INTRODUCTION}

Data reduction has always been widely researched due to limitations in storage space and communication bandwidth [1], [2]. The relevant issue in most such applications is to retain the meaning of the data as much as possible while reducing its size. This requirement led to several optimal methods, which employed self-organizing maps [3], ant colony [4], Fibonacci lattices [5], self growing and self organized neural gas [6], superposed histogram based adaptive clustering [7], dynamic programming [8], modified fuzzy $c$-means clustering [9], improved hard $c$-means clustering [10], and histogram-based $c$-means clustering [2]. Besides effective storage and communication, the most relevant applications of color reduction are in image segmentation [11],

Research supported by the Hungarian National Research Funds (OTKA), Project no. PD103921. The work of L. Kovács and S.M. Szilágyi was supported by the Hungarian Academy of Sciences through the János Bolyai Fellowship program.

${ }^{1}$ L. Szilágyi is with Dept. of Control Engineering and Information Technology, Budapest University of Technology and Economics, Magyar tudósok krt. 1, 1117 Budapest, Hungary (phone: +36-1-463-4027; fax: +361-463-2699) and with Dept. of Electrical Engineering, Sapientia University, Calea Sighişoarei 1/C, 540485 Tîrgu Mureş, Romania (phone: +40-265-206210; fax: +40-265-206-211; e-mail: lalo at ms.sapientia.ro).

${ }^{2}$ G. Dénesi is with Dept. of Electrical Engineering, Sapientia University, Calea Sighişoarei 1/C, 540485 Tîrgu Mureş, Romania (phone: +40265-206-210; fax: +40-265-206-211; e-mail: denesigellert at yahoo. com).

${ }^{3}$ L. Kovács is with Applied Informatics Institute, John von Neumann Faculty of Informatics, Obuda Univesity, Budapest, Hungary (e-mail: kovacs.levente at nik.uni-obuda.hu).

${ }^{4}$ S. M. Szilágyi is with Dept. of Informatics, Petru Maior Univesity, Str. N. Iorga nr. 1, 540088 Tîrgu Mureş, Romania (phone/fax: +40-265-262-275; e-mail: szsandor 72 at yahoo.com).
[12], [13] and document analysis [14].

In an earlier paper [2] we introduced a histogram-based formulation of the color reduction problem applicable for various $c$-means clustering algorithms. Input images were fed to a preliminary processing to extract and list the most frequently occurring colors, and then $c$-means clustering was applied to this list. This paper attempts to provide a comparative study of various fuzzy $c$-means algorithms with improved partition, based on their application for color reduction within the above mentioned framework. Algorithms involved in this study include generalized improved partition fuzzy $c$-means (GIFP-FCM) [15], [16], suppressed fuzzy $c$ means (s-FCM) [17], and various generalized versions of s-FCM [18]. Numerical analysis will be performed to reveal the accuracy and efficiency of the tested algorithms.

The rest of this paper is structured as follows. Section II presents the framework employed, while section III takes into account the algorithms involved in this study. Numerical evaluation is provided in section IV, while conclusions are given in the last section.

\section{THE FRAMEWORK}

In a previous paper [2] we introduced an efficient color reduction framework applicable with various clustering algorithms. The whole color reduction process was performed in three steps:

1) Preprocessing first provides a color quantization, reducing the possible colors from $2^{24} \approx 16.77$ millions to $52^{3} \approx 140$ thousands, followed by a color selection, which separates frequent quantized colors from rare ones via histogram thresholding, and feeds the set of frequent quantized colors to the next step.

2) Clustering applies the chosen version of $c$-means algorithm to the selected quantized colors and performs fast histogram-based [19] and memory-efficient [20] clustering. This process provides optimal cluster prototypes and a label to each selected quantized color.

3) Creating output image consists of labeling all existing quantized colors of the image and then repainting each pixel of the original image according to the label of the pixel' quantized color.

There are two main parameters in the framework, valid for all clustering algorithms:

1) The number of clusters $c$, which can vary between 2 and 256.

2) The selection percentage $P$ ranging $80-100 \%$ is the parameter of the color selection during preprocessing. 
It determines the amount of pixels that will count at the actual clustering. Excluding $10 \%$ of the pixels that belong to least frequent quantized color usually leaves outside of the selected set two thirds of the quantized colors [2].

Initial cluster prototypes are chosen as random selected colors constrained by a minimum difference criterion [21].

\section{EMPLOYED CLUSTERING ALGORITHMS}

\section{A. The conventional ones}

The conventional fuzzy $c$-means (FCM) algorithm partitions a set of object data $\mathbf{X}=\left\{\mathbf{x}_{1}, \mathbf{x}_{2}, \ldots, \mathbf{x}_{n}\right\}$ into a number of $c$ clusters based on the minimization of a quadratic objective function, defined as:

$$
J_{\mathrm{FCM}}=\sum_{i=1}^{c} \sum_{k=1}^{n} u_{i k}^{m}\left\|\mathbf{x}_{k}-\mathbf{v}_{i}\right\|_{A}^{2}=\sum_{i=1}^{c} \sum_{k=1}^{n} u_{i k}^{m} d_{i k}^{2},
$$

where $\mathbf{v}_{i}$ represents the prototype or centroid of cluster $i(i=1 \ldots c), u_{i k} \in[0,1]$ is the fuzzy membership function showing the degree to which vector $\mathbf{x}_{k}$ belongs to cluster $i, m>1$ is the fuzzyfication parameter, and $d_{i k}$ represents the distance (any inner product norm defined by a symmetrical positive definite matrix $A$ ) between $\mathbf{x}_{k}$ and $\mathbf{v}_{i}$. FCM uses a probabilistic partition, meaning that the fuzzy memberships assigned to any input vector $\mathbf{x}_{k}$ with respect to clusters satisfy the probability constraint $\sum_{i=1}^{c} u_{i k}=1$. The minimization of the objective function $J_{\mathrm{FCM}}$ is achieved by alternately applying the optimization of $J_{\mathrm{FCM}}$ over $\left\{u_{i k}\right\}$ with $\mathbf{v}_{i}$ fixed, $i=1 \ldots c$, and the optimization of $J_{\mathrm{FCM}}$ over $\left\{\mathbf{v}_{i}\right\}$ with $u_{i k}$ fixed, $i=1 \ldots c, k=1 \ldots n$ [22]. In each loop, the optimal values are deduced from the zero gradient conditions and Lagrange multipliers, and obtained as follows:

$$
\begin{gathered}
u_{i k}^{\star}=\frac{d_{i k}^{-2 /(m-1)}}{\sum_{j=1}^{c} d_{j k}^{-2 /(m-1)}} \quad \forall i=1 \ldots c, \forall k=1 \ldots n, \\
\mathbf{v}_{i}^{\star}=\frac{\sum_{k=1}^{n} u_{i k}^{m} \mathbf{x}_{k}}{\sum_{k=1}^{n} u_{i k}^{m}} \quad \forall i=1 \ldots c .
\end{gathered}
$$

According to the alternating optimization (AO) scheme of the FCM algorithm, Eqs. (2) and (3) are alternately applied, until cluster prototypes stabilize. This stopping criterion compares the sum of norms of the variations of the prototype vectors $\mathbf{v}_{i}$ within the latest iteration, with a predefined small threshold value $\varepsilon$.

Hard $c$-means [23] is a special case of FCM, which uses $m=1$, and thus the memberships are obtained by the winner-takes-all rule. Each cluster prototype will be the average of the input vectors assigned to the given cluster.

\section{B. Fuzzy c-means with improved partition}

Partitions provided by FCM have an undesired property: in the proximity of the boundary between two neighbor clusters, fuzzy memberships with respect to other clusters have local maxima, instead of being close to zero. To suppress this phenomenon without losing the fuzzy nature
TABLE I

SUPPRESSION RULES INCLUDED IN THIS STUDY, WHERE

$$
u_{w k}=\max \left\{u_{j k}, j=1 \ldots c\right\}
$$

\begin{tabular}{|c|c|}
\hline Suppression rule & Suppression rate formula \\
\hline $\mathrm{gs}_{\xi}$-FCM at $\xi=0.9$ & $\alpha_{k}=\frac{1-\left(\sin \frac{\pi u_{w k}}{2}\right)^{\xi}}{1-u_{w k}}$ \\
$\mathrm{gs}_{\tau}$-FCM at $\tau=0.5$ & $\alpha_{k}=\frac{1-\tau}{1+u_{w k} \tau}$ \\
$\mathrm{gs}_{\theta}-\mathrm{FCM}$ at $\theta=0.5$ & $\alpha_{k}=\left[1-u_{w k}+u_{w k}(1-\theta)^{2 /(1-m)}\right]^{-1}$ \\
\hline
\end{tabular}

of the algorithm, Höppner and Klawonn [15] introduced the so-called FCM with improved partition (IFP-FCM), which is derived from an objective function that additionally contains a rewarding term:

$$
J_{\mathrm{IFP}}=\sum_{i=1}^{c} \sum_{k=1}^{n} u_{i k}^{m} d_{i k}^{2}-\sum_{k=1}^{n} a_{k} \sum_{i=1}^{c}\left(u_{i k}-1 / 2\right)^{2},
$$

where parameters $a_{k}$ are positive numbers. The second term has the effect of pushing the fuzzy membership values $u_{i k}$, $i=1 \ldots c, k=1 \ldots n$ towards 0 or 1 , while maintaining the probabilistic constraint. Later, Zhu et al. [16] introduced a generalized version of this algorithm, derived from the objective function

$$
J_{\mathrm{GIFP}}=\sum_{i=1}^{c} \sum_{k=1}^{n} u_{i k}^{m} d_{i k}^{2}+\sum_{k=1}^{n} a_{k} \sum_{i=1}^{c} u_{i k}\left(1-u_{i k}^{m-1}\right),
$$

whose optimization leads to the partition update formula

$$
u_{i k}^{\star}=\frac{\left(d_{i k}^{2}-a_{k}\right)^{-1 /(m-1)}}{\sum_{j=1}^{c}\left(d_{j k}^{2}-a_{k}\right)^{-1 /(m-1)}} \quad \begin{aligned}
& \forall i=1 \ldots c, \\
& \forall k=1 \ldots n
\end{aligned} .
$$

Equation (6) explains us the behavior of GIFP-FCM: for any input vector $\mathbf{x}_{k}$, the square of its distances measured from all cluster prototypes are virtually reduced by a constant positive value $a_{k}$. The authors also proposed a formula for the choice of $a_{k}: a_{k}=\omega \min _{i}\left\{d_{i k}^{2}, i=1 \ldots c\right\}$, with $\omega \in$ $[0.9,0.99]$, thus keeping the square of all distorted distances positive. Using $\omega=1$ would reduce GIFP-FCM to HCM.

Both versions of the improved clustering models keep FCM's prototype update formula given in Eq. (3).

\section{Suppression of fuzzy c-means}

The suppressed fuzzy $c$-means (s-FCM) algorithm [17] had the declared goal of reducing the execution time of FCM by improving its convergence speed, while preserving its good partition quality. The s-FCM algorithm does not minimize $J_{\mathrm{FCM}}$.

Instead of that, it manipulates with the AO scheme of FCM, by inserting an extra computational step in each iteration, placed between the partition update formula (2) and prototype update formula (3). This new step deforms the partition (fuzzy membership functions) according to the following rule:

$$
\mu_{i k}=\left\{\begin{array}{ll}
1-\alpha+\alpha u_{i k} & \text { if } i=\arg \max _{j}\left\{u_{j k}\right\} \\
\alpha u_{i k} & \text { otherwise }
\end{array},\right.
$$


TABLE II

OVERALL ACCURACY RANKING BASED ON 300 RUNS (15 IMAGES $\times 20$ SCENARIOS). FIGURES INDICATE NUMBER OF TOP RANKED PERFORMANCES OUT OF 300

\begin{tabular}{|l|c|c|c|c|c|c|c|c|c|}
\cline { 3 - 10 } \multicolumn{1}{c|}{} & \multirow{2}{*}{ FCM } & \multicolumn{2}{c|}{ GIFP-FCM } & \multicolumn{2}{c|}{ s-FCM } & \multicolumn{3}{c|}{ gs-FCM } & HCM \\
\cline { 3 - 10 } \multicolumn{1}{c|}{} & & $\omega=0.9$ & $\omega=0.99$ & $\alpha=0.5$ & $\alpha=0.9$ & $\xi=0.9$ & $\tau=0.5$ & $\theta=0.5$ & \\
\hline Best & 14 & $\mathbf{1 3 7}$ & 11 & 18 & 73 & 26 & 5 & 13 & 3 \\
In top 2 & 35 & $\mathbf{1 7 6}$ & 48 & 53 & 123 & 74 & 35 & 50 & 6 \\
In top 3 & 46 & $\mathbf{2 0 3}$ & 67 & 99 & 163 & 126 & 84 & 98 & 14 \\
\hline Better than FCM & 251 & 210 & 240 & $\mathbf{2 8 0}$ & 258 & 233 & 242 & 84 \\
\hline
\end{tabular}

TABLE III

AVERAGE ACD VALUES OF 15 IMAGES OBTAINED IN 20 DIFFERENT SCENARIOS BY VARIOUS ALGORITHMS. BEST PERFORMANCE IS HIGHLIGHTED IN EACH SCENARIO

\begin{tabular}{|r|r||c|c|c|c|c|c|c|c|c|}
\hline$c$ & \multicolumn{1}{|c|}{$P$} & FCM & \multicolumn{2}{|c|}{ GIFP-FCM } & \multicolumn{2}{|c|}{ s-FCM } & \multicolumn{3}{|c|}{ gs-FCM } & HCM \\
\cline { 4 - 9 } & & $\omega=0.9$ & $\omega=0.99$ & $\alpha=0.5$ & $\alpha=0.9$ & $\xi=0.9$ & $\tau=0.5$ & $\theta=0.5$ & \\
\hline 16 & 100 & 7.5254 & 7.6518 & 7.7834 & 7.6012 & $\mathbf{7 . 4 4 5 7}$ & 7.5360 & 7.6233 & 7.6138 & 7.8251 \\
16 & 95 & 7.5838 & 7.6035 & 7.6476 & 7.5627 & $\mathbf{7 . 4 6 3 8}$ & 7.5435 & 7.5867 & 7.6314 & 7.6974 \\
16 & 90 & 7.7022 & 7.6058 & 7.6380 & 7.6181 & $\mathbf{7 . 5 7 1 4}$ & 7.6007 & 7.6061 & 7.6289 & 7.9226 \\
16 & 80 & 8.0188 & 7.7446 & 7.8232 & $\mathbf{7 . 7 2 0 0}$ & 7.7616 & 7.7527 & 7.7309 & 7.7361 & 8.1006 \\
\hline 32 & 100 & 5.6423 & 5.6355 & 5.8090 & 5.6244 & $\mathbf{5 . 4 9 8 1}$ & 5.5750 & 5.7042 & 5.6287 & 5.8590 \\
32 & 95 & 5.7185 & 5.5920 & 5.6873 & 5.5917 & $\mathbf{5 . 5 3 1 1}$ & 5.5567 & 5.6099 & 5.5957 & 5.9505 \\
32 & 90 & 5.8247 & $\mathbf{5 . 5 4 6 9}$ & 5.6436 & 5.5717 & 5.6209 & 5.5949 & 5.5861 & 5.5849 & 6.0411 \\
32 & 80 & 6.0848 & $\mathbf{5 . 7 9 3 9}$ & 5.8913 & 5.8314 & 5.8776 & 5.8611 & 5.8599 & 5.8190 & 6.7379 \\
\hline 64 & 100 & 4.3514 & 4.2656 & 4.4101 & 4.3228 & $\mathbf{4 . 1 9 7 5}$ & 4.2296 & 4.3406 & 4.2789 & 4.5330 \\
64 & 95 & 4.4281 & $\mathbf{4 . 1 9 7 7}$ & 4.2893 & 4.2558 & 4.2142 & 4.2119 & 4.2664 & 4.2347 & 4.6846 \\
64 & 90 & 4.5925 & $\mathbf{4 . 2 7 9 8}$ & 4.3473 & 4.3253 & 4.3149 & 4.3330 & 4.3334 & 4.3406 & 4.8910 \\
64 & 80 & 4.9793 & $\mathbf{4 . 4 7 9 4}$ & 4.5695 & 4.5647 & 4.6276 & 4.6285 & 4.5853 & 4.5933 & 5.4136 \\
\hline 128 & 100 & 3.5745 & 3.3784 & 3.5157 & 3.4431 & $\mathbf{3 . 3 3 3 7}$ & 3.3643 & 3.4643 & 3.3838 & 3.7372 \\
128 & 95 & 3.6116 & $\mathbf{3 . 3 6 3 6}$ & 3.4413 & 3.4120 & 3.3900 & 3.3982 & 3.4261 & 3.4110 & 3.8861 \\
128 & 90 & 3.7758 & $\mathbf{3 . 4 1 5 6}$ & 3.4906 & 3.4761 & 3.4985 & 3.5113 & 3.4817 & 3.4864 & 4.0708 \\
128 & 80 & 4.1226 & $\mathbf{3 . 7 2 3 4}$ & 3.7800 & 3.7769 & 3.8559 & 3.9156 & 3.7821 & 3.8683 & 4.5874 \\
\hline 256 & 100 & 3.0122 & $\mathbf{2 . 7 5 4 0}$ & 2.8967 & 2.8417 & 2.7614 & 2.8004 & 2.8514 & 2.7695 & 3.1139 \\
256 & 95 & 3.1168 & $\mathbf{2 . 7 6 8 3}$ & 2.8513 & 2.8202 & 2.8284 & 2.8858 & 2.8203 & 2.8203 & 3.3317 \\
256 & 90 & 3.2367 & $\mathbf{2 . 8 4 2 7}$ & 2.9075 & 2.8896 & 2.9412 & 3.0024 & 2.8907 & 2.9125 & 3.4750 \\
256 & 80 & 3.6879 & $\mathbf{3 . 1 4 1 4}$ & 3.1681 & 3.1906 & 3.3070 & 3.3646 & 3.1888 & 3.2632 & 4.0775 \\
\hline
\end{tabular}

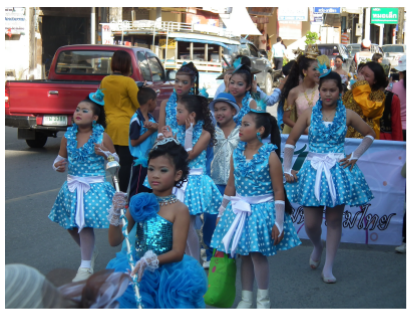

(a)

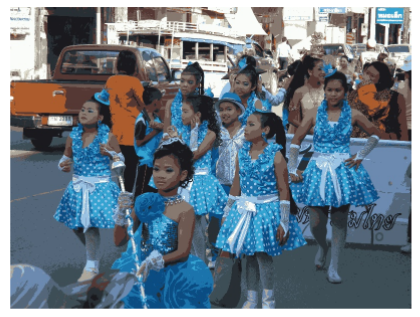

(b)

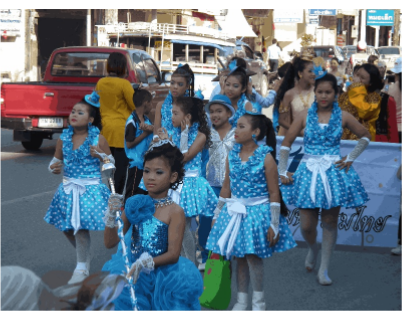

(c)

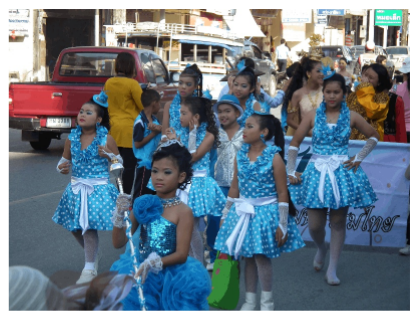

(d)

Fig. 1. Color reduction results of GIFP-FCM ( $\omega=0.9$ ): (a) original image; (b) 16 colors; (c) 64 colors; (d) 256 colors. Pixel selection rate was set to $P=90 \%$

TABLE IV

OVERALL EFFICIENCY RANKING BASED ON 300 RUNS ( 15 IMAGES $\times 20$ SCENARIOS). FIGURES INDICATE NUMBER OF TOP RANKED PERFORMANCES OUT OF 300

\begin{tabular}{|l|c|c|c|c|c|c|c|c|}
\cline { 3 - 8 } \multicolumn{1}{c|}{} & \multirow{2}{c|}{ FCM } & \multicolumn{2}{c|}{ GIFP-FCM } & \multicolumn{2}{c|}{ s-FCM } & \multicolumn{3}{c|}{ gs-FCM } \\
\cline { 3 - 9 } \multicolumn{1}{c|}{} & & $\omega=0.9$ & $\omega=0.99$ & $\alpha=0.5$ & $\alpha=0.9$ & $\xi=0.9$ & $\tau=0.5$ & $\theta=0.5$ \\
\hline Best & 30 & 3 & 57 & $\mathbf{1 0 7}$ & 4 & 1 & 77 & 21 \\
In top 2 & 48 & 9 & 97 & $\mathbf{1 9 9}$ & 12 & 6 & 181 & 48 \\
In top 3 & 77 & 12 & 167 & $\mathbf{2 5 2}$ & 23 & 22 & 239 & 108 \\
\hline Faster than FCM & 102 & 209 & 241 & 97 & 125 & $\mathbf{2 5 0}$ & 183 \\
\hline
\end{tabular}




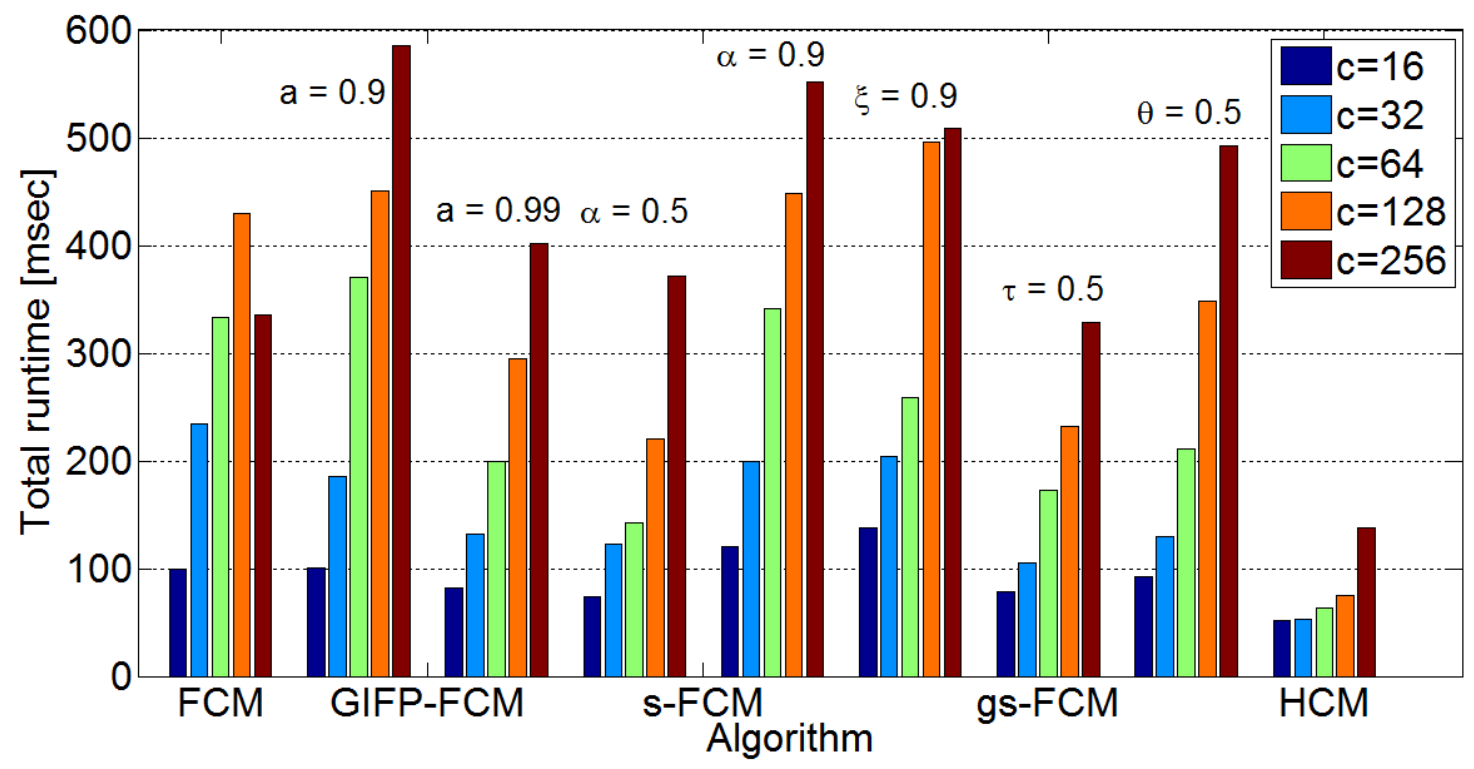

Fig. 2. Total runtime averaged over fifteen test images, plotted against applied algorithm and cluster number, at fixed pixel selection rate $P=90 \%$
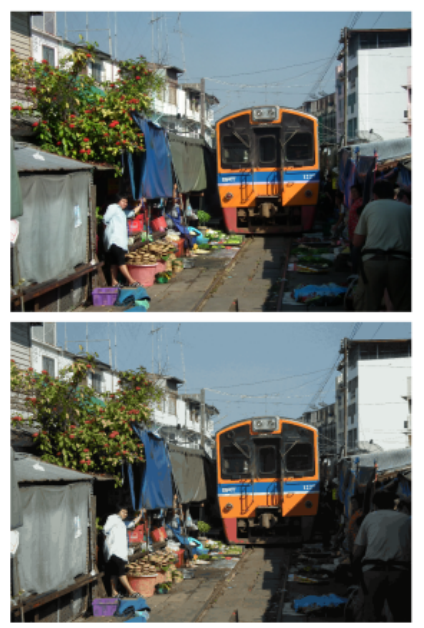

(a)
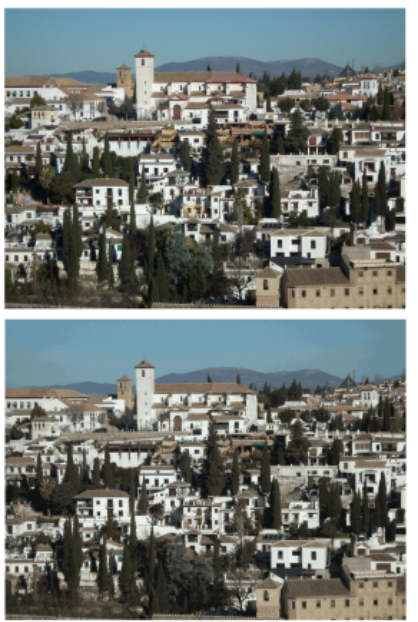

(e)

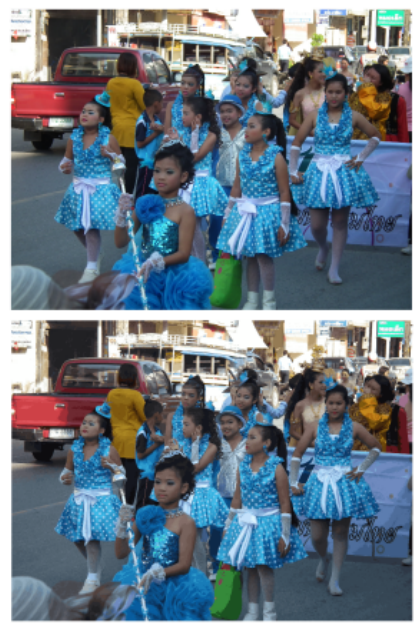

(b)
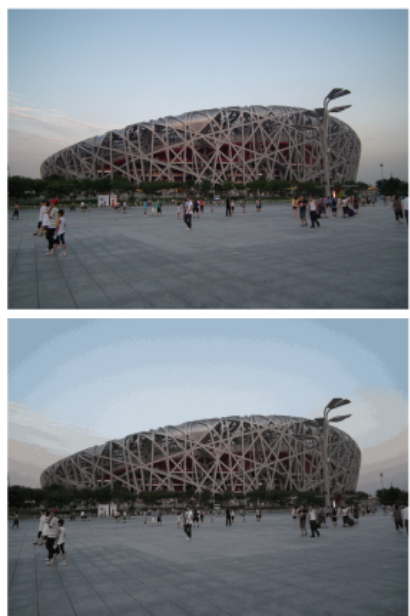

(f)
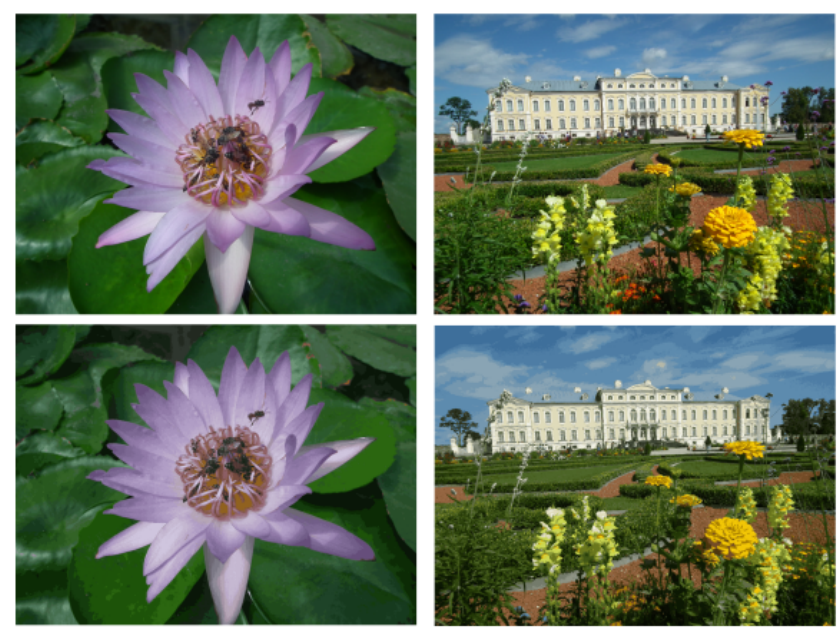

(c)
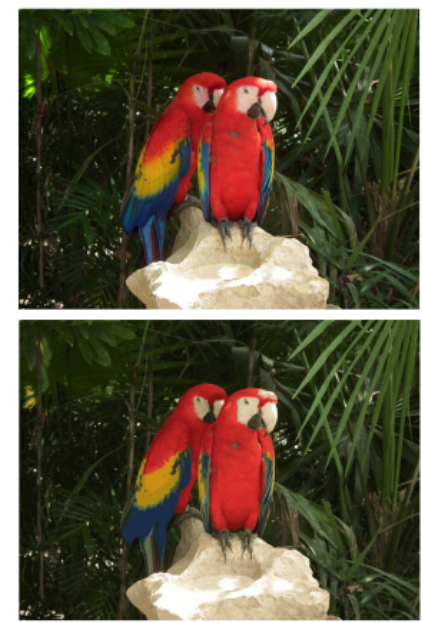

(g)

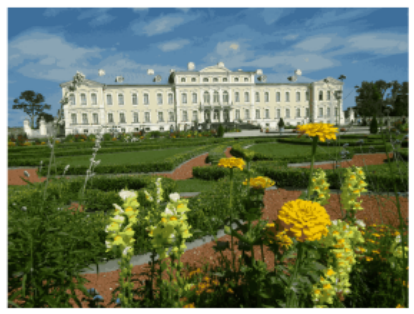

(d)
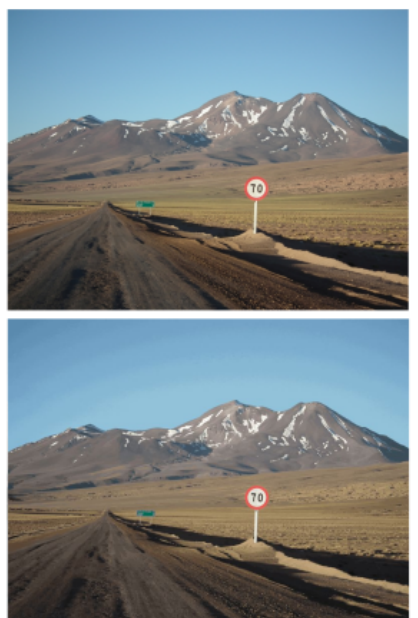

(h)

Fig. 3. Some images in original (upper row) and after color reduction (bottom row): 32 colors (c); 48 colors (b, e, f); 64 colors (d, g, h); 96 colors (a). 
where $\mu_{i k}(i=1 \ldots c, k=1 \ldots n)$ represents the fuzzy memberships obtained after suppression. During the iterations of s-FCM, these suppressed membership values $\mu_{i k}$ will replace $u_{i k}$ in Eq. (3). Suppression rate $\alpha=1$ makes s-FCM identical with FCM, while $\alpha=0$ with HCM. All other values of $\alpha$ lead to algorithms that differ from all above mentioned ones.

In an earlier paper [18] we have introduced several generalized rules for the suppression of the FCM partition, and showed the relation of all s-FCM versions with GIFP-FCM. These generalization rules consider the suppression rate context sensitive and thus assign a dedicated $\alpha_{k}$ suppression rate to each input vector $\mathbf{x}_{k}$ in each iteration. For this study, we have chosen some of the suppression rules found successful in [18], they are exhibited in Table I.

\section{NUMERICAL EVALUATION}

\section{A. Accuracy benchmarks}

To provide quantitative evaluation of the algorithms' accuracy, we will compute the averaged color distance (ACD) between the original input images and the final color reduced images in various scenarios, employing the formula:

$$
\mathrm{ACD}=\frac{1}{n} \sum_{k=1}^{n}\left\|\mathbf{x}_{k}-\mathbf{v}_{\lambda_{k}}\right\|
$$

where $\mathbf{x}_{k}$ is the color vector of pixel $k$, and $\lambda_{k}$ is the label given to pixel $k$.

Fifteen color photographs $(800 \times 600$ pixels, 8 -bit color depth) were fed to all algorithms presented in Section III, containing 3-30 thousand colors after color quantization. All algorithms were run at 20 different scenarios generated by parameters $c \in\{16,32,64,128,256\}$ and $P \in$ $\{80 \%, 90 \%, 95 \%, 100 \%\}$. Various benchmark indices were recorded, including $\mathrm{ACD}$, total runtime, and number of main loops performed. Table II exhibits the results of the 300 accuracy competitions (15 images $\times 20$ scenarios) of the nine algorithms. Although GIFP-FCM $(\omega=0.9)$ is ranked first, in best 2 , and in best 3 the most times, s-FCM $(\alpha=0.9)$ is the one that performs the most times better than FCM. Table III lists ACD values given by various algorithms in the 20 scenarios, averaged over the 15 images. Again here, GIFP-FCM $(\omega=0.9)$ and s-FCM share the first places. The former performs better for larger values of $c$ and lower values of $P$ parameter. Both tables show that HCM has the worst (but comparable) results out of all tested algorithms, and most improved partition algorithms indeed perform better than FCM in most cases.

Figure 1 shows an example of image used as input and three output images created by the GIFP-FCM algorithm, at $P=90 \%$ pixel inclusion rate. Sixteen colors proved definitely too few to produce acceptable images, although every relevant detail is visible also here. The other two representations show the output for $c=64$ and $c=256$ clusters. These images are of better quality. The latter can hardly be distinguished from the original, when screened or printed in small size. These images suggest that FCM-based algorithms are able to extract acceptable palettes of color images. Figure 3 exhibits several examples of reduced color images of good quality, which use only 32-96 colors.

\section{B. Efficiency benchmarks}

The efficiency of the algorithms was characterized by their total runtime including preprocessing and the creation of output image.

Table IV shows the results of the 300 efficiency competitions (15 images $\times 20$ scenarios) of the algorithms. HCM won almost all competitions, but it was excluded from this table as we were more interested in the competition of fuzzy ones. Here s-FCM $(\alpha=0.5)$ has top ranking the most times, but gs-FCM $(\tau=0.5)$ was found the one performing the most times quicker than FCM.

Figure 2 presents the average runtime of each tested algorithm, obtained in case of various cluster numbers $c$, and pixel selection rate $P=90 \%$. Averaging was performed over the fifteen test images. All efficiency tests were performed on a PC with i5 processor running at $2.8 \mathrm{GHz}$ frequency. Algorithms were allowed to perform up to 300 iterations unless convergence was reached. HCM was stopped after the first iteration that produced no change in labels, while all others used convergence threshold set to $\varepsilon=c / 10$.

\section{CONCLUSIONS}

In this paper we employed seven fuzzy $c$-means clustering algorithms with improved partition, together with HCM and FCM, in a color reduction problem, to provide a comparison in terms of accuracy and efficiency. All improved partition algorithms produced better accuracy than FCM, and four of them were found more efficient as well. As accuracy is the most important in color reduction, it is recommendable to choose GIFP-FCM using $\omega=0.9$. In applications where time also counts, gs-FCM with $\tau=0.5$ might be the right choice.

\section{REFERENCES}

[1] J. P. Braquelaire and L. Brun, "Comparison and optimization of methods of color image quantization," IEEE Transactions on Image Processing vol. 6, pp. 1048-1052, 1997.

[2] L. Szilágyi, G. Dénesi, and S. M. Szilágyi, "Fast color reduction using approximateive c-means clustering models," IEEE Int. Conf. Fuzzy Syst (FUZZ-IEEE), Beijing, accepted paper, 2014.

[3] J. Rasti, A. Monadjemi and A. Vafaei, "Color reduction using a multistage Kohonen self-organizing map with redundant features," Expert Systems with Applications, vol. 38, pp. 13188-13197, 2011.

[4] A. T. Ghanbarian, E. Kabir and N. M. Charkari, "Color reduction based on ant colony," Pattern Recognition Letters, vol. 28, pp. 1383-1390, 2007.

[5] A. Mojsilovic and E. Soljanin, "Color quantization and processing by Fibonacci lattices ," IEEE Transactions on Image Processing vol. 10, pp. 1712-1725, 2001.

[6] A. Atsalakis, and N. Papamarkos, "Color reduction and estimation of the number of dominant colors by using a self-growing and selforganized neural gas," Engineering Applications of Artificial Intelligence, vol. 19, pp. 769-786, 2006.

[7] I. S. Hsieh, K. C. Fan, "An adaptive clustering algorithm for color quantization," Pattern Recognition Letters, vol. 21, pp. 337-346, 2000.

[8] K. Kanjanawanishkul and B. Uyyanonvara, "Novel fast color reduction algorithm for time-constrained applications," Journal of Visual Communication and Image Representation vol. 16, pp. 311-332, 2005.

[9] D. Özdemir, and L. Akarun, "A fuzzy algorithm for color quantization of images," Pattern Recognition, vol. 35, pp. 1785-1791, 2002. 
[10] M. E. Celebi, "Improving the performance of $k$-means in color quantization," Image and Vision Computing, vol. 29, pp. 260-271, 2011.

[11] G. Dong and M. Xie, "Color clustering and learning for image segmentation based on neural networks," IEEE Transactions on Neural Networks vol. 16, pp. 925-936, 2005.

[12] M. Apró, D. Novalović, Sz. Pál, S. Dedijer and N. Milić, "Colour space selection for entropy-based image segmentation of folded substrate images," Acta Polytechnica Hungarica vol. 10, no. 1, pp. 43-62, 2013.

[13] F. Shi and P. McCauley Bush, "A Gaussian-mixed fuzzy clustering model on valence-arousal-related fMRI data-set," Acta Polytechnica Hungarica vol. 10, no. 8, pp. 85-104, 2013.

[14] N. Nikolaou, and N. Papamarkos, "Color reduction for complex document images," International Journal of Imaging Systems and Technologies, vol. 19, pp. 14-26, 2009.

[15] F. Höppner, and F. Klawonn, "Improved fuzzy partition for fuzzy regression models," Int. J. Approx. Reason., vol. 5, pp. 599-613, 2003

[16] Zhu, L., Chung, F. L., and Wang, S., "Generalized fuzzy $c$-means clustering algorithm with improved fuzzy partition," IEEE Trans. Syst. Man Cybern. B., vol. 39, pp. 578-591, 2009.

[17] J. L. Fan, W. Z. Zhen and W. X. Xie, "Suppressed fuzzy $c$-means clustering algorithm," Pattern Recognition Letters, vol. 24, pp. 1607$1612,2003$.

[18] L. Szilágyi, and S. M. Szilágyi, "Generalization rules for the suppressed fuzzy $c$-means clustering algorithm," Neurocomput., vol. 139, pp. 298-309, 2014.

[19] L. Szilágyi, Z. Benyó, S. M. Szilágyi and H. S. Adam, "MR brain image segmentation using an enhanced fuzzy $c$-means algorithm," Annual International Conference of IEEE EMBS, Cancún, pp. 724726, 2003.

[20] J. F. Kolen and T. Hutcheson, "Reducing the time complexity of the fuzzy c-means algorithm," IEEE Transactions on Fuzzy Systems vol. 10, pp. 263-267, 2002

[21] D. Arthur and S. Vassilvitskii, "k-means++: The advantages of careful seeding," Proc. 18th Ann. ACM-SIAM Symp. Discr. Alg., 2007, pp. $1027-1035$

[22] J. C. Bezdek, Pattern recognition with fuzzy objective function algorithms, New York: Plenum, 1981.

[23] H. Steinhaus, "Sur la division des corp materiels en parties", Bulletin de l'Academie Polonaise des Science, C1 III., vol. IV, pp. 801-804, 1956. 